\title{
ESTUDO DE EQUILÍBRIO E MODELAGEM CINÉTICA DA ADSORÇÃO DO CORANTE CRISTAL VIOLETA SOBRE CARVÃO DE CAPIM-ELEFANTE
}

\author{
R. R. M. da $\operatorname{SILVA}^{1}$, T. T. F. de OLIVEIRA $^{1}$ e A. B. FRANÇA ${ }^{1}$ \\ ${ }^{1}$ Universidade Federal de São João del-Rei, Departamento de Engenharia Química \\ E-mail para contato: robertaresende38@gmail.com
}

\begin{abstract}
RESUMO - A busca por adsorventes alternativos de baixo custo e eficientes na remoção de compostos catiônicos tem se intensificado nos últimos anos. Neste trabalho, o carvão foi produzido através da pirólise lenta do capim-elefante, sendo avaliada sua eficiência como adsorvente para a remoção do cristal violeta. Os modelos de cinética de pseudo-primeira ordem e pseudo-segunda ordem foram ajustados aos dados experimentais e a cinética de pseudo-segunda ordem descreveu a adsorção do corante sobre o adsorvente. Estudos de equilíbrio revelaram que o sistema foi melhor descrito termodinamicamente pela isortema Langmuir. A capacidade de adsorção máxima foi $15,601 \mathrm{mg} \cdot \mathrm{g}^{-1}$ e a porcentagem de remoção variou entre $73-95 \%$. Os resultados demonstraram que o carvão de capim-elefante é eficiente na remoção do corante cristal violeta.
\end{abstract}

\section{INTRODUÇÃO}

A remoção da cor dos efluentes é um dos grandes problemas enfrentados pelo setor têxtil. Estima-se que da quantidade total de corantes consumida pela indústria têxtil, cerca de $20 \%$ é descartada na forma de efluentes, sendo a principal fonte desta perda proveniente da incompleta fixação dos corantes durante a etapa de tingimento das fibras têxteis (Dallago et al., 2005).

A contaminação causada através do lançamento irregular deste tipo de efluente provoca a coloração dos cursos hidrícos e sérios danos à fauna e a flora locais, uma vez que essas substâncias restrigem a penetração dos raios solares, diminuindo a quantidade de oxigênio dissolvido na água e comprometendo a atividade fotossintética natural da biota aquática. Além disso, os corantes apresentam-se como recalcitrantes, o que dificulta sua degradação pelos sistemas de tratamento convencionais, e potencialmente cancerígenos (Freire e Freitas, 2010).

O processo de adsorção é um fenômeno caracterizado pela adesão de uma espécie química em estado líquido na superfície ou poros de um sólido, denominado adsorvente. A utilização de biomassa de resíduos na fabricação do carvão ativado, além de criar um destino apropriado e sustentável para os rejeitos industriais, apresenta-se como uma alternativa de baixo custo para o tratamento de efluentes contaminados por compostos orgânicos e catiônicos (Nunes, 2014). 
O capim-elefante (Pennisetum-purpureum Schaumacher) é uma espécie nativa da África, que foi introduzida e bem adaptada na maioria dos países subtropicais. Requer poucos nutrientes para seu crescimento e possui uma elavada taxa de produção de biomassa vegetal, podendo ser colhido até quatro vezes por ano (Strezov et al., 2008). O uso deste resíduo permitiria a redução da poluição ambiental, agregando valor à cultura do capim-elefante. Desta forma, este trabalho tem como objetivo investigar a viabilidade da aplicação do capimelefante para produção de carvão e como material adsorvente de corantes catiônicos.

\section{METODOLOGIA EXPERIMENTAL}

\subsection{Preparo do Adsorvente}

O capim-elefante in natura foi seco ao sol e posteriormente aquecido em estufa à $100^{\circ} \mathrm{C}$. Após a secagem, o capim foi picado em pedaços de aproximadamente $5 \mathrm{~cm} \mathrm{e}$ submetido á pirólise lenta durante 2 horas a $450{ }^{\circ} \mathrm{C}$, sob uma taxa de aquecimento de $10^{\circ} \mathrm{C} / \mathrm{min}$. O carvão obtido da carbonização foi cominuído através de um moinho de bolas (MARCONI). As partículas foram classificadas, segundo seu tamanho, utilizando-se um sistema de peneiras vibratórias (BERTEL Indústria Metalúrgica Ltda). As partículas com tamanho entre 12 e 32 mesh $(1,68$ e $0,5 \mathrm{~mm})$ foram selecionadas para a realização dos ensaios de adsorção. A Figura 1 ilustra o capim-elefante in natura e carvão de capim-elefante.

Figura 1- Capim-elefante (a) in natura; (b) carvão.

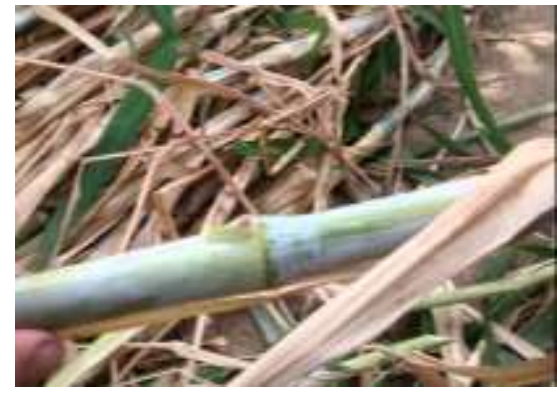

(a)

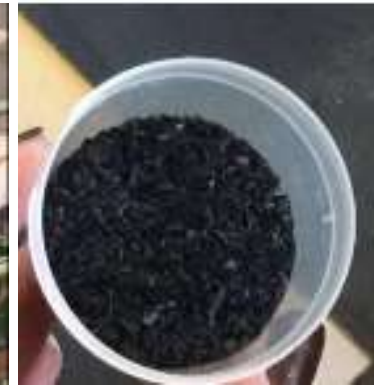

(b)

\subsection{Preparo do Efluente Sintético}

Preparou-se uma solução estoque $200 \mathrm{mg} . \mathrm{L}^{-1}$ de cristal violeta (Neon). Para a obtenção das soluções de concentrações menores, estas foram preparadas por diluição utilizando-se água destilada.

\subsection{Ensaios de Cinética e Isoterma}

Os estudos da cinética de adsorção foram realizados utilizando-se $5 \mathrm{~g}$ de carvão em 500 $\mathrm{mL}$ de solução contendo $180 \mathrm{mg} \cdot \mathrm{L}^{-1}$ do corante cristal violeta. Os ensaios foram feitos em batelada, em temperatura de $26^{\circ} \mathrm{C}$ e sob agitação constante de $200 \mathrm{rpm}$. A cada 20 minutos foram coletadas alíquotas da solução para medidas de concentração até que o equilíbrio fosse atingido. A análise da concentração do cristal violeta na solução foi realizada no comprimento de onda de $592 \mathrm{~nm}$ em um espectrofotômetro UV-Visível (Micronal-ASK 1600). 
Para obtenção das isotermas, foram preparadas cinco soluções de cristal violeta de concentrações iguais a 35, 70,160, 170 e $200 \mathrm{mg}$. $\mathrm{L}^{-1}$. As cinco amostras, contendo $250 \mathrm{ml}$ de solução e 0,25 g de carvão, foram submetidas à agitação em um shaker (TECNAL-TE-421). Após 180 minutos, foram recolhidas alíquotas de cada amostra, em triplicata, as quais foram submetidas a filtração, seguida de centrifugação por 5 minutos. Na sequência, as alíquotas foram quantificadas mediante a leitura da absorbância no espectrofotometro UV-Visível.

\section{RESULTADOS E DISCUSSÃO}

\subsection{Cinética de adsorção}

Na Figura 2 estão apresentadas as dependências da concentração do corante cristal violeta na solução em função do tempo.

Figura 2 - Concentração do corante na solução em função do tempo.

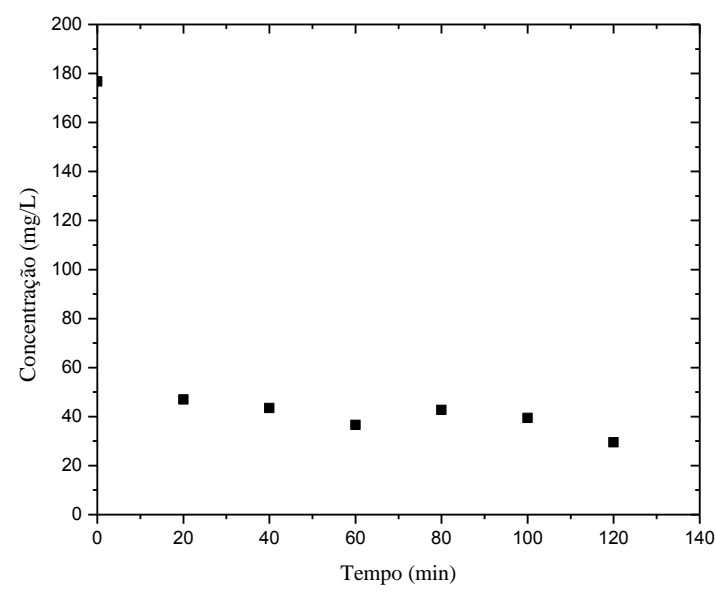

Como pode ser visto na Figura 2, obteve-se uma eficiência de remoção de cristal violeta de aproximadamente $73 \%$ em apenas 20 minutos de adsorção. Após o período de uma hora houve uma pequena oscilação na concentração devido a fraca dessorção em função de variações no pH (Annadurai et al., 2002), atingindo o equilíbrio após 120 minutos, com uma eficiência em cerca de $83 \%$ e um qe igual a $14,72 \mathrm{mg} \cdot \mathrm{g}^{-1}$.

A fim de compreender o mecanismo que rege o fenômeno de adsorção entre corante cristal violeta e carvão de capim-elefante, avaliou-se a cinética de adsorção descritas pelos modelos de pseudo-primeira e pseudo-segunda ordem (Ho e Mckay, 1999), definidos pelas Equações 1 e 2, respectivamente:

$$
q_{t}=q_{e}\left[1-e^{\left(-K_{1} \cdot t\right)}\right]
$$

onde $\mathrm{q}_{\mathrm{e}} \mathrm{e} \mathrm{q}_{\mathrm{t}}$ são as quantidades de corante adsorvidas $\left(\mathrm{mg} \cdot \mathrm{g}^{-1}\right)$ no equilíbrio e no tempo $\mathrm{t}$ (min), respectivamente, e $\mathrm{K}_{1}$ é a constante de velocidade de adsorção $\left(\mathrm{min}^{-1}\right)$, a qual pode ser obtida através da linearização da Equação 1, a partir da inclinação da reta do gráfico $\log \left(\mathrm{q}_{\mathrm{e}}{ }^{-}\right.$ $q_{t}$ ) versus $t$. 


$$
q_{t}=\frac{K_{2} \cdot q_{e}^{2} \cdot t}{1+\left(K_{2} \cdot q_{e^{-}} t\right)}
$$

onde $\mathrm{K}_{2}$ é a constante de velocidade de pseudo-segunda-ordem $\left(\mathrm{g} \cdot \mathrm{mg}^{-1} \cdot \mathrm{min}^{-1}\right)$, $\mathrm{q}_{\mathrm{e}}$ e $\mathrm{q}_{\mathrm{t}}$ são as quantidades de corante adsorvidas $\left(\mathrm{mg} \mathrm{g}^{-1}\right)$ no equilíbrio e no tempo $\mathrm{t}(\mathrm{min})$. A partir da reta do gráfico de $\mathrm{t} / \mathrm{q}_{\mathrm{t}}$ versus $\mathrm{t}$, o valor da constante $\mathrm{K}_{2}$ pode ser calculado.

A relação entre a quantidade adsorvida e o tempo está apresentada na Figura 3.

Figura 3 - Ajuste dos modelos cinéticos de pseudo-primeira e pseudo-segunda ordem aos dados experimentais da adsorção do cristal violeta em carvão de capim-elefante.

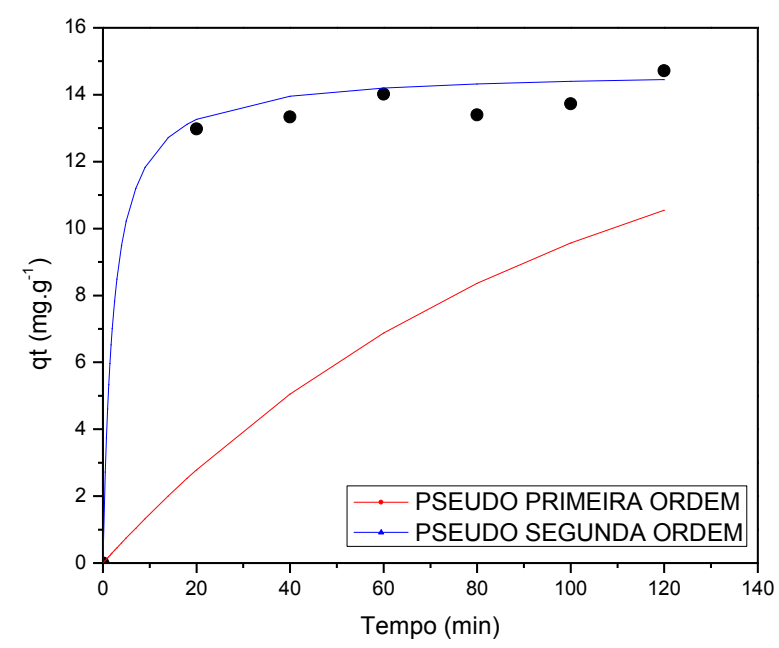

A partir dos coeficientes de correlação $\left(\mathrm{R}^{2}\right)$, e pelos valores de $\mathrm{q}_{\mathrm{e}}$ calculados com o ajuste dos modelos e de $q_{\mathrm{e}}$ obtidos a partir dos resultados experimentais, para ambos os modelos, conclui-se que os dados apresentaram melhor ajuste ao modelo de pseudo-segunda ordem, no qual obteve-se um $\mathrm{q}_{\mathrm{e}}$ igual a $14,39 \mathrm{mg} \cdot \mathrm{g}^{-1}$, muito próximo ao valor experimental $\left(\mathrm{q}_{\mathrm{e}(\exp )}=14,72 \mathrm{mg} \cdot \mathrm{g}^{-1}\right)$ e um coeficiente de correlação de 0,995 . Sugerindo uma adsorção química entre o carvão de capim-elefante e o corante violeta cristal, com possível formação de complexos ou grupos superficiais devido ao mecanismo de pareamento iônico (Ho e Mckay, 1999). Este tipo de mecanismo é caracterizado como praticamente irreversível, o que afeta a possibilidade de reutilização do adsorvente.

\subsection{Isoterma de adsorção}

A isoterma de adsorção foi utilizada para indicar como as moléculas adsorvidas de cristal violeta se distribuem entre a fase líquida e as partículas de adsorvente quando o processo atinge o estado de equilíbrio. A análise dos dados experimentais através do ajuste de diferentes modelos de isotermas é um passo importante para melhor compreensão do mecanismo do processo e de como o adsorvente e o adsorvato interagem (Zhu et al., 2009). Os modelos de Langmuir e Freundlich foram adequados aos dados de equlíbrio do sistema carvão de capim-elefante e cristal violeta, conforme demonstra a Figura 4. 
Figura 4 - Ajuste dos modelos de Langmuir e de Freundlich aos dados experimentais da adsorção entre o cristal violeta e o carvão de capim-elefante.

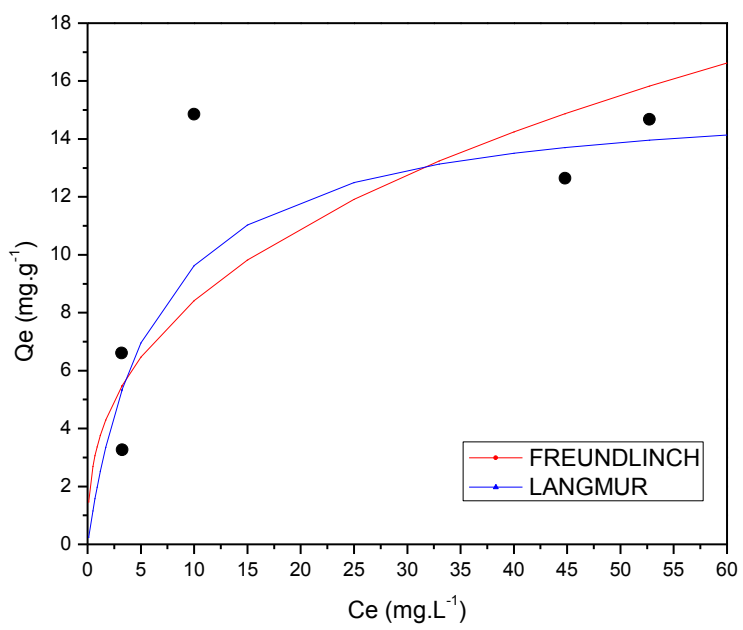

A Tabela 1 apresenta os valores para os parâmetros ajustados das isotermas de adsorção de Langmuir e Freundlich, respectivamente.

Tabela 1 - Parâmetros das isotermas de Langmuir e Freundlich

\begin{tabular}{|c|c|c|c|}
\hline \multicolumn{2}{|c|}{ Langmuir } & \multicolumn{2}{c|}{ Freundlich } \\
\hline $\mathrm{q}_{\text {máx }}\left(\mathrm{mg} \cdot \mathrm{g}^{-1}\right)$ & 15,601 & $\mathrm{n}$ & 2,633 \\
\hline $\mathrm{b}\left(\mathrm{L} \cdot \mathrm{mg}^{-1}\right)$ & 0,161 & $\mathrm{~K}_{\mathrm{F}}\left(\left(\mathrm{mg} \cdot \mathrm{g}^{-1}\right) \cdot\left(\mathrm{L} \cdot \mathrm{mg}^{-1}\right)^{1 / \mathrm{n}}\right)$ & 3,510 \\
\hline $\mathrm{R}^{2}$ & 0,945 & $\mathrm{R}^{2}$ & 0,618 \\
\hline
\end{tabular}

Os resultados apresentados na Tabela 1 e na Figura 4 demonstraram que a isoterma de Langmuir foi o modelo que melhor se ajustou aos dados experimentais, apresentando um coeficiente de correlação igual a 0,945, ou seja, a adsorção ocorre em monocamadas e os adsorvatos são adsorvidos quimicamente em número fixo e definido de sítios (Brandão, 2006). Provavelmente, a isoterma de Freundlich não representa de forma adequada o processo, por considerar a heterogeneidade da superfície do material adsorvente e não prever a saturação dos sítios. Houve uma redução na concentração inicial de corante em todas as amostras, nas quais a porcentagem de remoção variou entre $73-95 \%$.

As características essenciais da equação de Langmuir podem ser expressas em função de um termo adimensional conhecido como fator de separação $\left(\mathrm{R}_{\mathrm{L}}\right)$, definido pela Equação 3:

$$
R_{L}=\frac{1}{1+b \cdot C_{0}}
$$

onde $\mathrm{C}_{0}$ corresponde a concentração do soluto com maior concentração inicial (mg.L- $\left.{ }^{1}\right)$, b é a constante de adsorção de Langmuir $\left(\mathrm{L}_{\mathrm{mg}}{ }^{-1}\right)$. O valor do fator de separação diz se o processo de adsorção é desfavorável $\left(\mathrm{R}_{\mathrm{L}}>1\right)$, linear $\left(\mathrm{R}_{\mathrm{L}}=1\right)$, favorável $\left(0<\mathrm{R}_{\mathrm{L}}<1\right)$, ou irreversível $\left(\mathrm{R}_{\mathrm{L}}=0\right.$ ) ( Dotto et al., 2011). Para este sistema foi encontrado um valor de $\mathrm{R}_{\mathrm{L}}$ igual a 0,03 , o que indica um processo de adsorção favorável. 


\section{CONCLUSÕES}

Os resultados mostraram que o sistema corante cristal violeta e carvão de capimelefante foi melhor descrito cineticamente pelo modelo de pseudo-segunda ordem, por apresentar o maior coeficiente de correlação, assim como os menores desvios entre a quantidade adsorvida calculada e experimental no equilíbrio. A isoterma de Langmuir foi o modelo que melhor se ajustou aos dados experimentais, caracterizado pela adsorção química em monocamadas, o que também foi verificado através dos testes cinéticos. $\mathrm{O}$ carvão de capim-elefante apresenta-se como um adsorvente alternativo de baixo custo com potencial para o tratamento de soluções aquosas contaminadas por corantes catiônicos. Para trabalhos futuros sugere-se que seja feita a caracterização deste material.

\section{REFERÊNCIAS}

ANNADURAI, G.; JUANG, R. S.; LEE, D. J. Use of cellulose-based wastes for adsorption of dyes from aqueous solutions. J. of Hazard. Mater., v. 92, n. 3, p. 263-274, 2002.

BRANDÃO, P.C. Avaliação do uso do bagaço de cana como adsorvente para remoção de contaminantes derivados do petróleo de efluentes. Uberlândia: Dissertação de mestrado. Programa de Pós-Graduação em Engenharia Química da Universidade Federal de Uberlândia, 2006. 147 p.

DAllaGo, R. M., SMANiOTTO, A., OliVEIRA, L. D. Resíduos sólidos de curtumes como adsorventes para a remoção de corantes em meio aquoso. Quím. Nova, v. 28, p. 433-437, 2005.

DOTTO, G. L., VIEIRA, M. L. G., GONÇALVES, J. O., PINTO, L. A. A., Removal of acid blue 9, food yellow 3 and FD\&C yellow $\mathrm{n}^{\circ} 5$ dyes from aqueous solutions using activated carbon, activated earth, diatomaceous earth, chitin and chitosan: Equilibrium studies and thermodynamic. Quím. Nova, v. 34, p. 1193-1199, 2011.

FREIRE, F. B.; FREITAS, S. L. DE. Avaliação da remoção de cor de um efluente têxtil sintético. Eng. Amb., v. 7, n. 3, p. 241-249, 2010.

HO, Y. S.; MCKAY, G. Pseudo-second order model for sorption processes. Process Biochem., v. 34, pg. 451-465, 1999.

NUNES A. S.; Produção de adsorventes a partir da casca de amendoim visando a aplicação na remoção de corantes orgânicos. Itapetinga: Dissertação de Mestrado. Programa de PósGraduação em Ciências Ambientais da Universidade Estadual do Sudoeste da Bahia, Itapetinga, 2014. 66p.

STREZOV, V., EVANS, T. J., HAYMAN, C. Thermal conversion of elephant grass Pennisetum Purpureum Schum) to bio-gas, bio-oil and charcoal. Bioresour. Technol., v. 99, n. 17, p. 8394-8399, 2008.

ZHU C., WANG L., CHEN W. Removal of Cu (II) from aqueous solution by agricultural byproduct: peanut hull. J. of Hazard. Mater., vol. 168, p. 739-746, 2009. 